\title{
How many episodes of hospital care might be prevented by widespread uptake of pneumococcal conjugate vaccine?
}

\section{E D G Mclntosh}

Correspondence to: Dr E D G Mclntosh, Wyeth, Huntercombe Lane South, Taplow, Maidenhead, Berkshire SL6 OPH; UK; mcintod@wyeth.com

Accepted

5 February 2003
Background: It is likely that disease specific infectious morbidity is under-reported. Microbiologically identifiable diseases may be "hidden" in ICD-10 code as "unspecified" disease.

Aims: To estimate the proportion of "unspecified" morbidity of infectious cause in infants and young children reported by Hospital Episode Statistics (HES) in England in 1999 that could reasonably be attributed to Streptococcus pneumoniae, and to calculate what number and proportion of diseases could potentially be prevented by a programme of pneumococcal conjugate vaccination.

Methods: Proportions of HES "unspecified" septicaemia, meningitis, and pneumonia attributable to pneumococcal infection were estimated by applying theoretical rates obtained from studies using highly sensitive diagnostic tests. The numbers obtained were added to those coded as pneumococcal in origin. The vaccine preventable proportion was then calculated using serogroup coverage, disease specific efficacy, and vaccine uptake.

Results: For infants and children 3 months to 5 years of age in 1999, HES reported 134, 245, and 216 episodes of pneumococcal septicaemia, meningitis, and pneumonia respectively. In addition, 68, 36 , and 2548 episodes of "unspecified" disease respectively are probably pneumococcal in origin. For hospitalisations in England in this age group, 157/202 (78\%) cases of pneumococcal septicaemia, $218 / 281(76 \%)$ cases of pneumococcal meningitis, and 452/2764 (16\%) cases of pneumococcal pneumonia may be preventable annually by means of pneumococcal conjugate vaccination.

Conclusions: Paediatric hospital morbidity in England due to pneumococcal septicaemia, meningitis, and pneumonia is under-reported by $34 \%, 13 \%$ and $92 \%$ respectively. A larger proportion of morbidity is preventable than implied by ICD-10 code alone.
$T$ he application of sensitive molecular tests such as the polymerase chain reaction (PCR) to the diagnosis of infectious diseases may gain wider acceptance with the publication of the Department of Health document Getting ahead of the curve. ${ }^{1}$ Application of these tests can lead to the inclusion of a proportion of cases of otherwise "unspecified" infection being assigned a definitive microbiological diagnosis. This approach has recently been applied theoretically to pneumococcal infection where the actual mortality rate due to invasive pneumococcal disease (IPD) for the year 1999 in infants and children 1 month to 4 years of age in England and Wales was found to be 43 deaths per year, around three times the published rate. ${ }^{2}$ The number of deaths in that age group potentially preventable with 7-valent pneumococcal conjugate vaccine (PCV) was found to be 26 per year $(60 \%)$, the same number as prevented with Haemophilus influenzae type b (Hib) vaccine. A US study based on the Active Bacterial Core Surveillance and the National Vital Statistics System for the period 1996-98 concluded that IPD deaths in all age groups may be underestimated by between $15 \%$ and $45 \%{ }^{3}$.

The present study examines the possibility that, in addition to mortality, pneumococcal morbidity is under-represented in published data, in this case in Hospital Episode Statistics (HES). It also calculates what proportion and number of cases of pneumococcal septicaemia, meningitis, and pneumonia could potentially be prevented by 7-valent PCV.

\section{METHODS}

HES data are compiled from over 300 NHS authorities in England; the most recent primary episode data available, for the year 1999, were used. The age group analysed was 3 months to 5 years. It was assumed that each period of care under a consultant represents a distinct episode of disease in an individual patient. Infants and children suffering multiple episodes of septicaemia, meningitis, and pneumonia were assumed to be in a minority. ${ }^{4}$

A full description of the theoretical basis for this methodology is to be found in the related "mortality study", with the exception that a lower rate ( $12.5 \%$ versus $45 \%)$ of "unspecified" meningitis potentially due to Streptococcus pneumoniae has been utilised. Briefly, PCR, latex agglutination, and other alternate methodologies to microbiological culture have been applied in various studies to the detection of Streptococcus pneumoniae in paediatric patients. ${ }^{5-7}$ The authors of these studies applied such tests to subjects with culture negative "unspecified" septicaemia, meningitis, or pneumonia, thereby estimating the proportion possibly due to pneumococcal infection (table 1). In the present analysis the implications of these tests, mainly pneumolysin PCR, were applied theoretically to the "unspecified" morbidity due to septicaemia, meningitis, and pneumonia reported by the HES.

By applying the same theoretical model used in the "mortality study", it was assumed that $16 \%$ of "unspecified" septicaemia, ${ }^{5} 12.5 \%$ of "unspecified" meningitis, ${ }^{6}$ and $36 \%$ of "unspecified" pneumonia ${ }^{7}$ is pneumococcal in origin. These

Abbreviations: HES, Hospital Episode Statistics; Hib, Haemophilus influenzae type b; IPD, invasive pneumococcal disease; PCV, pneumococcal conjugate vaccine; PCR, polymerase chain reaction 
Table 1 Summary of sensitivity and specificity of PCR, latex agglutination, and other tests in the detection of pneumococcal septicaemia, meningitis, and pneumonia, and the application of these tests to "unspecified" septicaemia, meningitis, and pneumonia in the cited studies

\begin{tabular}{llll}
\hline Clinical syndrome & $\begin{array}{l}\text { Zhang et al }(1995)^{5} \\
n=36, \text { Septicaemia }\end{array}$ & $\begin{array}{l}\text { Berkley et al }(2001)^{6} \\
n=905, \text { Meningitis }\end{array}$ & $\begin{array}{l}\text { Toikka et al }(1999)^{7} \\
n=90, \text { Pneumonia }\end{array}$ \\
\hline Sensitivity & $4 / 5(80 \%)$ & $28 / 29(96.6 \%)$ & $3 / 4(75 \%)$ \\
Specificity & $26 / 31(84 \%)$ & $427 / 434(98.4 \%)$ & $6 / 6(100 \%)$ \\
"Unspecified" disease & $5 / 31(16 \%)$ & $1 / 8(12.5 \%)$ & $14 / 39(36 \%)$ \\
\hline
\end{tabular}

proportions were reduced by $20 \%$ to account for those who may be coincidental nasopharyngeal carriers of Streptococcus pneumoniae. ${ }^{8}$ HES data were gathered by ICD-10 code as follows: pneumococcal septicaemia, meningitis, and pneumonia-A403, G001, and J13 respectively; "unspecified" septicaemia, meningitis, and pneumonia-A419, G009/G039, and J180/J181/J188/J 189 respectively.

The preventable proportion of 1999 pneumococcal morbidity was then calculated on the basis of $85.8 \%$ serogroup coverage, ${ }^{9}$ a 7 -valent PCV efficacy of $97.4 \%$ for IPD $^{10}$ an efficacy of $20.5 \%$ for pneumococcal pneumonia, ${ }^{11}$ and a vaccine uptake of $93 \% .^{12}$

\section{RESULTS}

Table 2 shows the results. For infants and children aged 3 months to 5 years in England for the HES reporting period 1999, there were 379 hospitalised cases coded as pneumococcal septicaemia and meningitis, and 216 episodes coded as pneumococcal pneumonia. If more sensitive diagnostic tests had been applied, an additional 104 cases of "unspecified" septicaemia and meningitis, and 2548 cases of "unspecified" pneumonia would probably be assigned pneumococcal aetiology. Thus, morbidity in England resulting from pneumococcal septicaemia, meningitis, and pneumonia may be underestimated by $34 \%, 13 \%$, and $92 \%$ respectively. While, in particular, pneumococcal septicaemia is underestimated by
$49 \%$ in the age group 3-5 months and pneumococcal meningitis is underestimated by $33 \%$ in the age group 3-5 years, pneumococcal pneumonia is underestimated in all age groups. Of the 8853 cases of "unspecified" pneumonia in the age group 3 months to 5 years, 5317 (60\%) are coded J181 lobar pneumonia.

Table 2 also shows the proportion of pneumococcal morbidity that may be preventable contingent on a universal immunisation programme with 7-valent PCV. The greatest impact of such a programme would be on the age group 3-5 months where the model predicts a $37 \%$ reduction in morbidity. In all the age groups this reduction is $20 \%$ or greater. For meningitis in the age group 3 months to 5 years, 218 cases would be prevented yearly in England alone which, at 14 days per admission represents not only around 3000 hospital inpatient days saved, but a considerable burden of sequelae prevented.

\section{DISCUSSION}

The major contributor to this analysis for estimating the likely morbidity burden of pneumococcal disease in England is pneumonia. This reflects the lack of microbiological precision in diagnosing pneumonia. By contrast the considerable underestimate of pneumococcal septicaemia is in infants 3-5 months of age and of pneumococcal meningitis is in children 3-5 years of age. In the former the empiric use of antibiotics is

Table 2 Pneumococcal and "unspecified" septicaemia, meningitis, and pneumonia by age group 3 months to 5 years; number of hospital episodes in England in 1999 and number potentially preventable with 7-valent PCV

\begin{tabular}{|c|c|c|c|c|c|c|c|}
\hline & $3-5 \mathrm{mth}$ & $6-11 \mathrm{mth}$ & $1 y$ & $2 y$ & $3 y$ & $4-5 y$ & Total \\
\hline \multicolumn{8}{|l|}{ Confirmed pneumococcal (p) } \\
\hline Septicaemia & 17 & 40 & 41 & 24 & 4 & 8 & 134 \\
\hline Meningitis & 53 & 98 & 47 & 31 & 8 & 8 & 245 \\
\hline Pneumonia & 21 & 41 & 68 & 33 & 27 & 26 & 216 \\
\hline Totals (p) & 91 & 179 & 156 & 88 & 39 & 42 & 595 \\
\hline \multicolumn{8}{|c|}{ Number of "unspecified" cases possibly due to $S$ pneumococus $(u) /$ total number of "unspecified" cases ( $t$ ) } \\
\hline Septicaemia & $16 / 124$ & $18 / 142$ & $16 / 123$ & $8 / 60$ & $4 / 36$ & $6 / 52$ & $68 / 537$ \\
\hline Meningitis & $10 / 105$ & $8 / 78$ & $6 / 53$ & $4 / 40$ & $3 / 32$ & $5 / 44$ & $36 / 352$ \\
\hline Pneumonia & $170 / 593$ & $421 / 1462$ & $739 / 2567$ & $495 / 1720$ & $325 / 1127$ & $398 / 1384$ & $2548 / 8853$ \\
\hline Totals (U) & 196 & 447 & 761 & 507 & 332 & 409 & 2652 \\
\hline \multicolumn{8}{|l|}{ Subtotals $(p+u)$} \\
\hline Septicaemia & 33 & 58 & 57 & 32 & 8 & 14 & 202 \\
\hline Meningitis & 63 & 106 & 53 & 35 & 11 & 13 & 281 \\
\hline Pneumonia & 191 & 462 & 807 & 528 & 352 & 424 & 2764 \\
\hline Total $(p+u)$ & 287 & 626 & 917 & 595 & 371 & 451 & 3247 \\
\hline \multicolumn{8}{|l|}{ Subtotals preventable with 7 -valent PCV* } \\
\hline Septicaemia & 26 & 45 & 44 & 25 & 6 & 11 & $157(78 \%)$ \\
\hline Meningitis & 49 & 82 & 41 & 27 & 9 & 10 & $218(78 \%)$ \\
\hline Pneumonia & 31 & 76 & 132 & 86 & 58 & 69 & $452(16 \%)$ \\
\hline Totals preventable with 7-valent PCV (\%) & $106(37)$ & $203(32)$ & $217(24)$ & $138(23)$ & $73(20)$ & $90(20)$ & $827(26 \%)$ \\
\hline
\end{tabular}

*Preventable $=(p+u) \times$ serogroup coverage $\times$ vaccine efficacy $\times$ vaccine uptake

For septicaemia and meningitis: $(p+u) \times 0.858 \times 0.974 \times 0.93$

For pneumonia: $(p+u) \times 0.858 \times 0.205 \times 0.93$. 
the most likely explanation, while in the latter it is probably missed or delayed diagnosis due to the insidious clinical presentation of pneumococcal meningitis.

The main source of microbiological data for pneumococcal morbidity comes from the Public Health Laboratory Service, ${ }^{913}$ although there are no data provided on "unspecified" disease and therefore limited potential to calculate the extent of under-reporting. Enhanced surveillance in England and Wales is one possibility for calculating this, ${ }^{14}$ and a national register is another. ${ }^{15}$ The whole UK population is around 20\% greater than that for England alone, which would mean that the UK hospital episodes in this age group would be around 3900 and the preventable number would be around 1000. It is possible that the extent of under-reporting extends not only throughout the hospital system but also in general practice, where cases of pneumonia are seen without referral to a paediatrician.

The studies applying alternate tests to "unspecified" disease $e^{5-7}$ first seek to establish the sensitivity and specificity of the test in infants and children with known disease or absence of disease confirmed by blood culture. They then apply the test to infants and children who are suspected of having disease but are culture negative. The study by Zhang and colleagues ${ }^{5}$ was performed in the USA, while that by Berkley and colleagues $^{6}$ was performed in Africa and that by Toikka and colleagues $^{7}$ was performed in Finland. It is possible that the rates of PCR positivity are different in the UK.

When comparing the present analysis with that in the pneumococcal mortality paper, ${ }^{2}$ it should be noted that two independent data sets were utilised, the Office for National Statistics in the former and HES in the latter. In the former, 43 deaths were described: five due to septicaemia, 13 due to meningitis, and 25 due to pneumonia. It is not clear precisely where these infants and children died. Although there may be some overlap, the effect on the morbidity numbers, 202, 281, and 2764 respectively, would be minimal. However, assuming that all the deaths were in hospital, this translates into case fatality rates of $2.5 \%, 4.9 \%$, and $0.9 \%$ for pneumococcal septicaemia, meningitis, and pneumonia respectively.

Recently, 7-valent PCV has been recommended for certain at-risk groups in the $\mathrm{UK}^{15}$ although the majority of cases of pneumococal infection occur in infants and children without recognised risk factors. A universal programme of infant and early childhood 7-valent PCV immunisation could lower paediatric pneumococcal morbidity in the UK. At least 827 courses of antibiotics may be prevented in England alone, and maybe more, given the possibility of a generalised decreased use of antibiotics in the era of widespread infant and early childhood pneumococcal immunisation. ${ }^{16}$ Assuming an average length of admission of 7 days for septicaemia, 14 days for meningitis, and 2 days for pneumonia, this also represents around 5055 preventable inpatient days.

Until specimen collection and transport is optimised, and molecular techniques are applied more widely, a number of infections such as those caused by Streptococcus pneumoniae, and other pathogens such as Mycobacterium tuberculosis and hepatitis B virus, will remain under-reported and "hidden" in code.

\section{CONCLUSIONS}

(1) Microbiologically identifiable diseases may be "hidden" in ICD-10 code.
(2) Further studies are needed in the UK using pneumolysin PCR assays in order to identify clearly the number of pneumococcal infections that are really "hidden" in ICD-10 code.

(3) Morbidity in England resulting from pneumococcal septicaemia, meningitis, and pneumonia may be underestimated by $34 \%, 13 \%$, and $92 \%$ respectively

(4) A larger proportion of morbidity due to septicaemia, meningitis, and pneumonia is preventable than that implied by ICD-10 code alone. For hospitalised cases in England in infants and children 3 months to 5 years of age, 157/202 (78\%) cases of pneumococcal septicaemia, 218/281 (76\%) cases of pneumococcal meningitis, and 452/2764 (16\%) cases of pneumococcal pneumonia may be prevented by means of a programme of universal infant 7-valent PCV immunisation.

\section{ACKNOWLEDGEMENTS}

We acknowledge: Carly Windsor, HES Project, IBM Basingstoke; and Zaf Hussain, NHS Information Authority, Birmingham.

Competing interest: The author is employed by Wyeth, which owns and markets 7-valent PCV.

\section{Author's affiliation}

E D G Mclntosh, Senior Medical Adviser, Wyeth Vaccines, and Honorary Clinical Senior Lecturer, Faculty of Medicine, Imperial College, London, UK

\section{REFERENCES}

1 DoH. Getting ahead of the curve. Department of Health, 2001. http://www.doh.gov.uk/cmo/idstrategy/index.htm

2 Mclntosh EDG, Booy R. Invasive pneumococcal disease in England and Wales: what is the true burden and what is the potential for prevention using 7 valent pneumococcal conjugate vaccine? Arch Dis Child 2002;86:403-6.

3 Moore MR, Gamble ML, Zell ER, et al. Deaths due to invasive Streptococcus pneumoniae, United States 1996-1998 (abstract 875). 39 th Annual Meeting of the Infectious Diseases Society of America, 25-28 October 2001, San Francisco.

4 Turett GS, Blum S, Telzak EE. Recurrent pneumococcal bacteremia: risk factors and outcomes. Arch Intern Med 2001;161:2141-4.

5 Zhang Y, Isaacman DJ, Wadowsky RM, et al. Detection of Streptococcus pneumoniae in whole blood by PCR. J Clin Microbiol 1995;33:596601

6 Berkley JA, Mwangi IM, Ngetsa CJ, et al. Diagnosis of acute bacterial meningitis in children at a district hospital in sub-Saharan Africa. Lancet 2001:357:1753-7.

7 Toikka P, Nikkari S, Ruuskanen O, et al. Pneumolysin PCR-based diagnosis of invasive pneumococcal infection in children. J Clin Microbiol 1999;37:633-7.

8 Dagan R, Shriker O, Hazan I, Leibovitz E, et al. Prospective study to determine clinical relevance of detection of pneumococcal DNA in sera of children by PCR. J Clin Microbiol 1998;36:669-73.

9 George RC, Melegaro A. Invasive pneumococcal infection in England and Wales 1999. CDR Weekly 2001;11(21).

10 Black S, Shinefield H, Fireman B, et al, and the North California Kaiser Permanente Vaccine Study Center Group. Efficacy, safety and immunogenicity of heptavalent pneumococcal conjugate vaccine in children. Pediatr Infect Dis J 2000;1 19:187-95

11 Black S, Shinefield PR, Ling S, et al. Effectiveness of heptavalent pneumococcal conjugate vaccine in children younger than five years of age for prevention of pneumonia. Pediatr Infect Dis J 2002;21:810-15.

12 http://www.phls.org.uk/topics az/vaccination/cover.htm.

13 Miller E, Waight $P$, Efstratiou A, et al. Epidemiology of invasive and other pneumococcal disease in children in England and Wales 1996-1998. Acta Paediatr 2000; (suppl 435):11-16.

14 Sleeman K, Knox K, George R, et al. Invasive pneumococcal disease in England and Wales: vaccination implications. J Infect Dis 2001;183:239-46.

15 Finn A, Booy R, Moxon R, et al. Should the new pneumococcal vaccine be used in high-risk children? Arch Dis Child 2002;87:18-21.

16 Lee GM, Fleisher GR, Harper MB. Management of febrile children in the age of conjugate vaccine: a cost-effectiveness analysis. Pediatrics 2001;108:835-44. 